\title{
Status of the SoLid experiment at the BR2 reactor
}

\author{
lanthe Michiels*广 \\ Ghent University \\ E-mail: ianthe.michielseugent.be
}

\begin{abstract}
The observation of the Reactor Antineutrino Anomaly, at the beginning of this decade, has revived the interest in short-baseline experiments that probe the disappearance of electron antineutrinos. In addition, the recent evidence for a distortion in the reactor antineutrino energy spectrum, seen by some of those short-baseline experiments, has questioned our current models even more.

The SoLid experiment is a reactor neutrino experiment, located at the BR2 reactor site of the $\mathrm{SCK} \bullet \mathrm{CEN}$ in Belgium, that aims to resolve the anomaly and perform a precise spectral measurement using a novel segemented detector design.

The first phase of SoLid has reached one year of data taking and the experiment will soon be able to present some initial results. These proceedings summarise the current status of the experiment.
\end{abstract}

European Physical Society Conference on High Energy Physics - EPS-HEP2019 -

10-17 July, 2019

Ghent, Belgium

\footnotetext{
* Speaker.

${ }^{\dagger}$ on behalf of the SoLid Collaboration
} 


\section{Introduction}

The observation of the Reactor Antineutrino Anomaly [1], at the beginning of this decade, has revived the interest in short-baseline experiments that probe the disappearance of electron antineutrinos. In addition, the recent evidence for a distortion in the reactor antineutrino energy spectrum, seen by some of those short-baseline experiments [2, 3, 4], has questioned our current models even more. The "Search for oscillations with a ${ }^{6}$ Lithium detector" (SoLid) experiment is a reactor neutrino experiment that will probe the reactor anomaly deficit and search for oscillation using a novel detector design based on highly segmented plastic scintillator. Installed at a very short distance of $\sim 6$ - $10 \mathrm{~m}$ from the BR2 research reactor at SCK $\bullet$ CEN in Belgium, it will be able to search for sterile neutrino oscillations through the detection of low energy $\overline{v_{e}}$. It will also exploit the high purity in ${ }^{235} \mathrm{U}$ of the BR2 reactor fuel, to increase our knowledge on reactor flux models and trace the origin of the spectral distortion.

\section{The SoLid detection principle and $B R 2$ reactor site}

The SoLid experiment uses a new segmented detector technology based on small detection cells that consist of a cube of polyvinyl-toluene (PVT) scintillator for the detection of electromagnetic (EM) interactions and two thin sheets of ${ }^{6} \mathrm{LiF}: \mathrm{ZnS}(\mathrm{Ag})$ for neutron capture. These cells are individually wrapped in optically isolating Tyvek paper and instrumented with wavelength-shifting fibre arrays that transport the scintillation signals to Multi-Pixel Photon Counters (MPPCs) for the read out.

Figure 1 illustrates how a reactor antineutrino interacts in part of the detector volume, resulting in an inverse beta decay (IBD) reaction in which a positron and a neutron are created. The positron annihilates in the PVT, giving a fast and sharp scintillation pulse; the neutron first thermalises by scattering through the detector material and is then captured by the ${ }^{6} \mathrm{Li}$ in the neutron detection sheets. This neutron capture by ${ }^{6} \mathrm{Li}$ creates an energetic $\alpha$ particle and triton $\left({ }^{3} \mathrm{H}\right)$, which excite the inorganic $\mathrm{ZnS}(\mathrm{Ag})$ scintillator. The combination of the prompt positron signal and a delayed series of decay pulses from the ${ }^{6} \mathrm{LiF}: \mathrm{ZnS}$ mixture builds up the antineutrino signature, with a characteristic time difference $\Delta t \sim 60 \mu \mathrm{s}$. For more details on the SoLid detection principle, one can consult Ref. [5]. The selection of IBD events is based on the time correlation between positron and neutron capture, their spatial correlation due to high detector segmentation, and on pulse-shape discrimination of the scintillation signals.

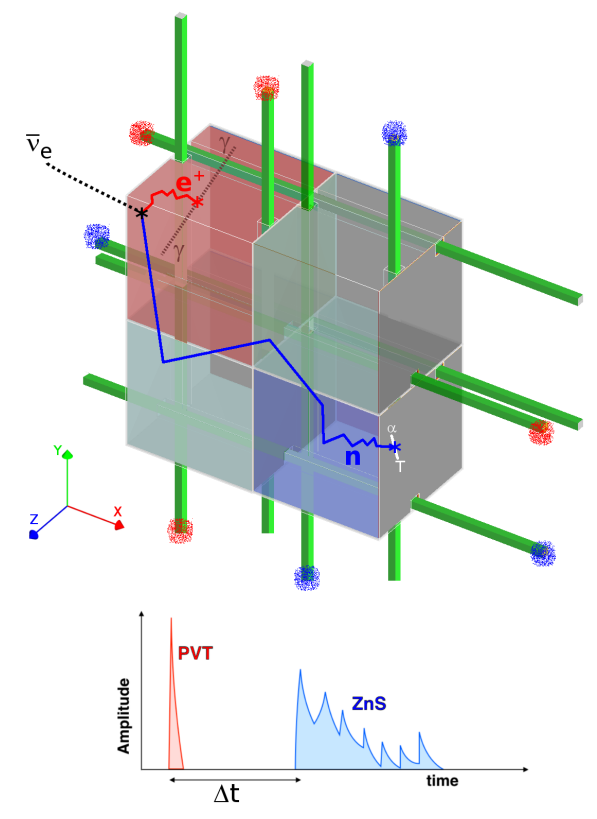

Figure 1: Schematic view of an antineutrino interaction with part of the SoLid detector (top). The resulting IBD signature is also sketched (bottom). 
Between the end of 2016 and the spring of 2018 the full scale SoLid Phase 1 detector was built and was then commissioned and installed at the BR2 reactor site in Mol, Belgium. The detector consists of 50 vertical planes, each composed of $16 \times 16$ detection cells of $(5 \times 5 \times 5) \mathrm{cm}^{3}$, resulting in a total sensitive mass of $1.6 \mathrm{t}$. The detector planes are grouped per 10 in a so called detector module, and each module is placed on a rail system in a container. This container is cooled to about $10{ }^{\circ} \mathrm{C}$ and is surrounded by additional $(50 \mathrm{~cm})$ shielding using slabs of HDPE on the top and bottom and walls of water bricks on the sides. It is also instrumented with a remotely operated calibration system, called CROSS [6].

As illustrated in figure 2, the SoLid set-up is positioned on-axis with the BR2 reactor core and its point of closest approach is 6.2 meters. The compact research reactor has a core of $\sim 50 \mathrm{~cm}$ diameter and is highly enriched $(93.5 \%)$ in ${ }^{235} \mathrm{U}$. BR2 operates in cycles of ca. 24 days with a power between 50 and $80 \mathrm{MW}$.

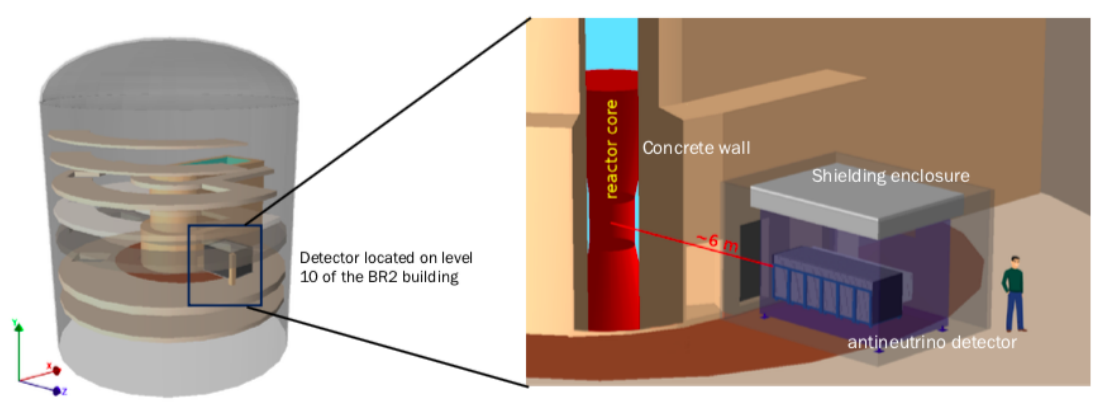

Figure 2: Schematic of the SoLid Phase 1 detector at the BR2 reactor site.

\section{Data reconstruction}

Three different triggers are implemented in the SoLid read-out system; a neutron trigger, which uses a pulse shape discrimination (PSD) algorithm, a threshold trigger, which is based on signal coincidences in crossing $\mathrm{X}$ and $\mathrm{Y}$ fibres with energies above $2 \mathrm{MeV}$, and a random trigger that reads out the full detector with a rate of $1 \mathrm{~Hz}$ [7]. The waveforms that pass the trigger are processed offline and are first clustered together in time. In a second step, the cluster lengths and PSD parameters are analysed to divide them into three possible categories; nuclear signals (NS), electromagnetic signals (ES) or muon signals. Examples of the clustered waveforms corresponding to an ES and NS signal are shown in figure 3.

\section{Backgrounds}

Given its location near a nuclear reactor, the SoLid detector site is prone to a lot of reactor induced backgrounds, such as gamma-rays and environmental neutrons. Apart from that, since the detector site has very low overburden ( $\sim 10$ m.w.e.), the experiment is also sensitive to cosmic and atmospheric backgrounds. One of the largest background contributions comes from fast neutrons from muon spallation or atmospheric showers that enter the detector and create recoils on Hydrogen. These recoils mimic the prompt ES and the neutrons themselves are moderated and 

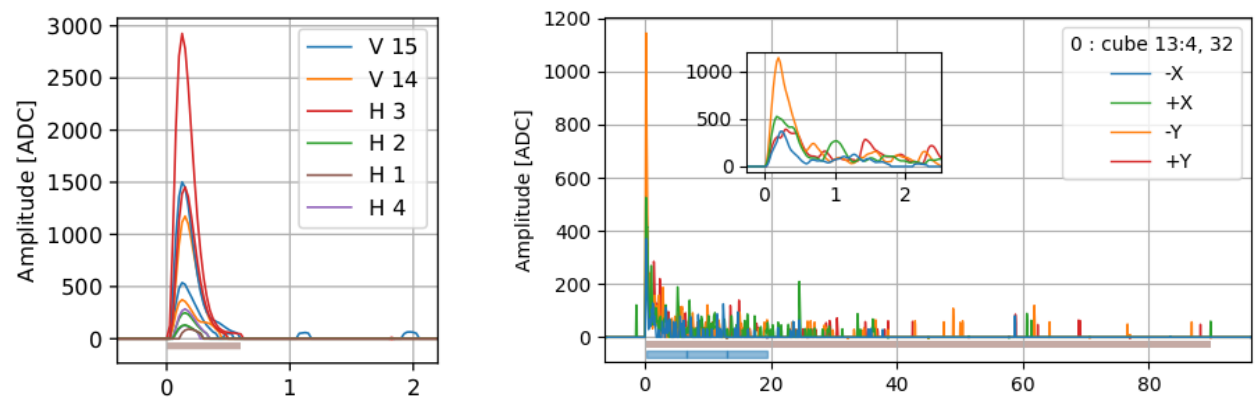

Figure 3: Example of a cluster of ES waveforms (left) and NS waveforms (right). Both x-axes show the time in $\mu$ s.

then detected as the delayed NS.

Another main experimental background is an internal one, related to the contamination of the ${ }^{6} \mathrm{LiF}: \mathrm{ZnS}(\mathrm{Ag})$ screens with elements from the ${ }^{238} \mathrm{U} /{ }^{232} \mathrm{Th}$ series. This series contains the decay from ${ }^{214} \mathrm{Bi}$ to ${ }^{214} \mathrm{Po}$ to ${ }^{210} \mathrm{~Pb}$, inducing the subsequent emission of a $\beta^{-}$and $\alpha$-particle. The $\beta^{-}$ particle has an ES signature, the $\alpha$ interacts with the $\mathrm{ZnS}(\mathrm{Ag})$ and, like neutrons captured on ${ }^{6} \mathrm{Li}$, induces a NS signal. Given the half-life of Po of $164 \mu$ s and the typical time difference between the prompt and delayed signal for fast neutron recoils of $\sim 70 \mu \mathrm{s}$, it is clear why these correlated backgrounds are hard to discriminate from the IBD processes which have an ES-NS time difference of $\sim 60 \mu \mathrm{s}$.

The SoLid collaboration is exploring various techniques to discriminate the IBD signal from the different types of (un)correlated backgrounds, some of which are described in the following section.

\section{IBD selection cuts}

To select a pure sample of antineutrino induced IBD events with an efficient set of selection cuts, the SoLid collaboration is developing a range of techniques and determining the most discriminating parameters. The set of cuts currently includes

- a muon veto, excluding all events recorded in a certain time span after a muon signal,

- an NS multiplicity veto, excluding all NS events recorded within a short time from each other,

- $\Delta x, \Delta y, \Delta z$ topological cuts between the ES and NS event,

- energy limits on the prompt signal: $1.5 \mathrm{MeV}<\operatorname{energy(ES)~}<20 \mathrm{MeV}$,

- $0<\Delta t<150 \mu \mathrm{s}$ as allowed detection time difference between the ES and NS events,

- a fiducial cut on the outer detector layer,

- an energy balance and BiPonisher cut.

As mentioned before, one of the dominant backgrounds arises from the subsequent decay of Bi and Po, where an energetic $\alpha$-particle mimics the IBD neutron detection. The energy balance 
and BiPonisher cuts are developed to reduce this background and are explained here in a bit more detail. The BiPonisher cut is a pulse shape discrimination algorithm to discriminate the neutron and $\alpha$ interaction in $\mathrm{ZnS}$. It is based on the difference in the $\alpha$ 's energy, which is lower in the ${ }^{6} \mathrm{Li}$ break-up in the IBD process, since that releases only $4.78 \mathrm{MeV}$, compared to the $7.69 \mathrm{MeV}$ that is released in the Po decay. This translates to a difference in the $\alpha$ 's waveform integral, as can be seen when comparing calibration data taken with an AmBe neutron source and a BiPo selection from reactor off data (cf. figure 4).

The energy balance cut is using the difference in energy balance between the two detector cubes that show the largest energy deposits for the prompt ES signal. Figure 5 shows this balance for BiPo background and the IBD signal; a cut at ES2/ES1=0.3 can significantly reduce the BiPo background, whilst retaining most of the IBD events.
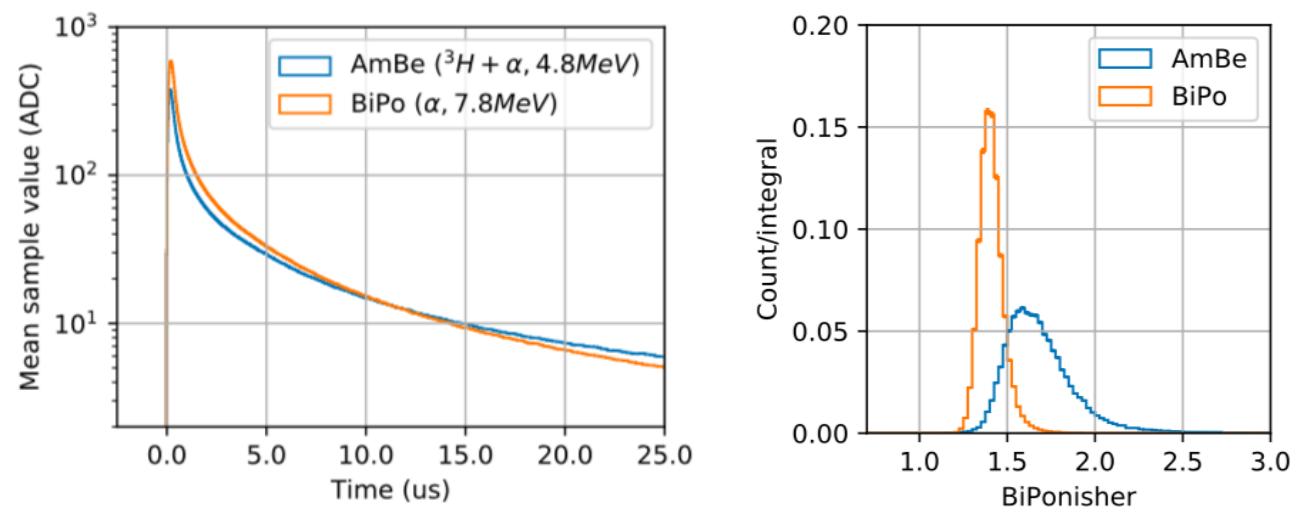

Figure 4: Illustration of the input (left) and discrimination power (right) of the PSD algorithm "BiPonisher" used to distriminate $\alpha$ from neutron interaction in the $\mathrm{ZnS}$ scintillator.
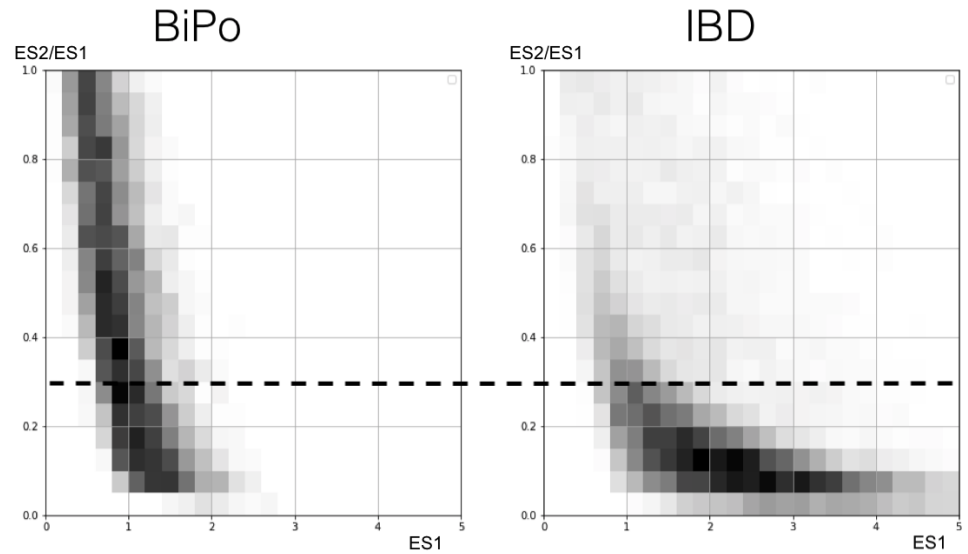

Figure 5: The energy balance between the detector cubes containing the two largest energy deposits from a prompt ES signal for BiPo events (left) and IBD Monte Carlo events (right).

The described IBD selection requirements are under development at the time of writing. These cuts result in an IBD selection efficiency of $\sim 15 \%$, which will be further improved by fine-tuning 
some of the cuts described and/or by using more advanced discrimination techniques. Given a predicted signal based on Monte Carlo of 1087 IBD interactions/day, using the current IBD efficiency of $15 \%$ and a background shape and rate based on 7.28 days of reactor off data, a preliminary signal-to-background ratio as a function of reconstructed ES energy is shown in figure 6.

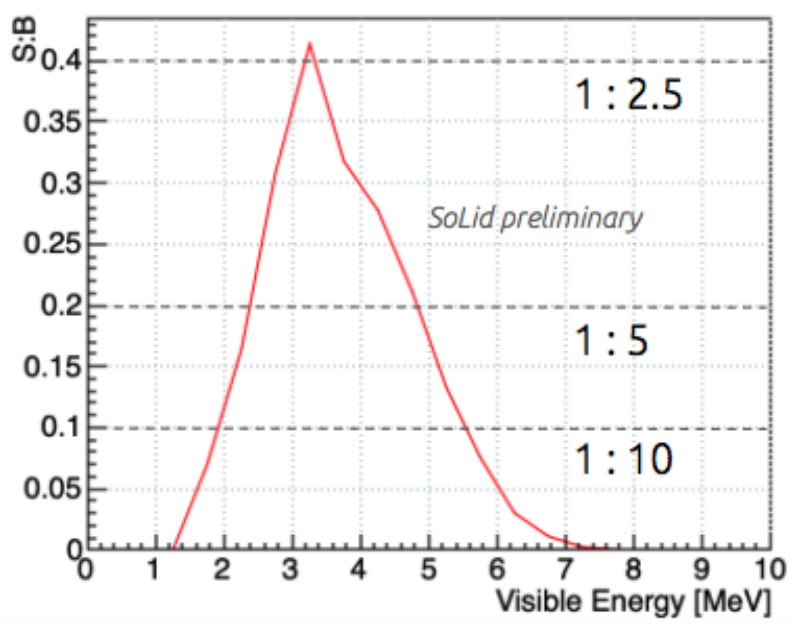

Figure 6: Current status of the signal-to-background ratio as a function of reconstructed ES energy. The ratio exceeds $1: 10$ in the energy region of interest.

\section{Outlook}

The SoLid experiment is making good progress in developing algorithms for object reconstruction as well as IBD selection requirements. The data analysis is ongoing and focuses on topological information for background reduction. A further improvement on the $\mathrm{S}: \mathrm{B}$ figure is expected when using a lower energy threshold and improving the IBD selection requirements. The sterile neutrino oscillation analysis is under development and will soon be applied to the data gathered during 6 reactor cycles and 1 year of detector operation.

\section{References}

[1] G. Mention et al., The reactor antineutrino anomaly, Phys. Rev. D 83073006 (2011)

[2] Double CHOOZ collaboration, Y. Abe et al., Improved measurements of the neutrino mixing angle $\theta_{13}$ with the Double CHOOZ detector, JHEP 10 (2014) 086

[3] Daya Bay collaboration, F.P. An et al., Measurement of the reactor antineutrino flux and spectrum at Daya Bay, Phys. Rev. Lett. 116 (2016) 061801

[4] RENO collaboration, J.H. Choi et al., Observation of energy and baseline dependent reactor antineutrino disappearance in the RENO experiment, Phys. Rev. Lett. 116 (2016) 211801

[5] SoLid Collaboration, Y. Abreu et al., A novel segmented-scintillator antineutrino detector, JINST 12 04 (2017) 
[6] L. Manzanillas for the SoLid Collaboration, Performance of the SoLid reactor neutrino detector, these proceedings

[7] SoLid Collaboration, Y. Abreu et al., Commissioning and Operation of the Readout System for the SoLid Neutrino Detector, arXiv:1812.05425 (2018) 